\title{
Zum Erscheinen des 150. Bandes der Ophthalmologica
}

In diesem Jahr 1965 erscheint der 150. Band einer wissen-schaftliehen Zeitschrift, die 1899 unter dem Namen «Zeitschrift fur Augenheilkunde» zum erstenmal herausgekommen ist. Begründer der Zeitschrift waren H. Kuhnt und /. von Michel, die in S. Karger (Berlin) nicht nur einen Verleger voller Verständnis fanden, sondern auch einen Menschen, der das Glück gehabt hat, in seinem Sohn Heinz und seinem Enkel Thomas Karger Nachfolger zu haben, die bestrebt waren, die Zeitschrift auf einem hohen Niveau zu halten. Bald errang sich die «Zeitschrift für Augenheilkunde» ein hohes Ansehen nicht nur in Deutschland, sondern auch in vielen anderen Ländern. Als Nachfolger der Gründer übernahmen J.Meller (seit 1922) und C.Behr (seit 1925) die Redaktion. Die Umstände, welche die Übersiedelung des Verlages Karger von Berlin nach Basel veranlaßten, führten zur Umwandlung der deut-schen Zeitschrift in eine Internationale: Sie bekam den Namen OPHTHALMOLOGİCA und publizierte Arbeiten in deutscher, eng-lischer und französischer Sprache. Die Redaktion wurde im Jahre 1938 von A. Bruckner (Basel) und H. J. M. Weve (Utrecht) über-nommen; ersterer stellt ihr auch heute noch, trotz seiner 88 Jahre, seine Erfahrung zur Verfügung.

Die Umgestaltung der Zeitschrift sollte dazu dienen, über die sprachlichen und politischen Grenzen hinweg den Austausch wissen-

3

schaftlicher Gedanken zu fördern und den Forschern Gelegenheit zu geben, die Ergebnisse ihrer Arbeit einem möglichst großen Kreis von Fachkollegen zu unterbreiten. Mit Befriedigung dürfen wir feststellen, daß die OPHTHALMOLOGICA ihr Ziel erreicht hat. Sie ist zu einem in alien Ländern des Nordens und Südens, des Ostens und Westens in einer Vielzahl von Exemplaren verbreiteten Organ intensiven geistigen Austauschs und freundschaftlicher Zu-sammenarbeit von Ophthalmologen aus aller Welt geworden. Auch \vährend der schwierigen Jahre wurden Arbeiten von Autoren aus den unterdrückten Ländern aufgenommen, die sonst kaum die Möglichkeit gehabt hätten, zu publizieren.

Die Auswahl der Manuskripte bleibt eine unserer größten Auf-gaben. Wir bedauern sehr, daß wir mitunter interessante Aufsätze wegen ihres zu großen Umfanges zurückweisen müssen, da wir uns an den Grundsatz halten, nur möglichst kurze, aber konzentrierte Arbeiten zu bringen.

Seit Jahren ist OPHTHALMOLOGICA auch das offizielle Publikationsorgan der Schweizerischen und der Niederländischen Ophthalmologischen Gesellschaft.

Das hohe Ansehen, das sich OPHTHALMOLOGICA in vielen Jahren erworben hat, verpflichtet. Es wird deshalb das Bemühen der Redaktion und des Verlages sein, die Zeitschrift immer weiter zu verbessern und für die Leser wertvoller zu gestalten.

Oktober 1965 E. B. Streíff

Pour la parution du 150ème volume de Ophthalmologica

En cette année 1965 paraît le 150ème volume d'une revue scien-tifique, qui a été publiée pour la premiere fois en 1899 sous le titre « Zeitschrift für Augenheilkunde » - « Journal

d'Ophtalmologie ».

L. Les fondateurs de la revue étaient H. Kuhnt et/. von Michel 
qui trouvèrent en S. Karger (Berlin) non seulement un éditeur com-préhensif, mais encore un horame qui eut la chance d'avoir comme successeurs son fils Heinz et son petit-fils Thomas Karger qui s'efforcèrent de maintenir le journal à un niveau élevé. Le « Jour- 